\title{
Scale Bridging Modeling of Plastic Deformation and Damage Initiation in Polycrystals
}

\author{
Anxin Ma and Alexander Hartmaier \\ Interdisciplinary Centre for Advanced Materials Simulation, Ruhr-University Bochum
}

Germany

\section{Introduction}

Plastic deformation of polycrystalline materials includes dislocation slip, twinning, grain boundary sliding and eigenstrain produced by phase transformations and diffusion. These mechanisms are often alternative and competing in different loading conditions described by stress level, strain rate and temperature. Modelling of plasticity in polycrystalline materials has a clear multiscale character, such that plastic deformation has been widely studied on the macro-scale by the finite element methods, on the meso-scale by representative volume element approaches, on the micro-scale by dislocation dynamics methods and on the atomic scale by molecular dynamics simulations. Advancement and further improvement of the reliability of macro-scale constitutive models is expected to originate from developments at microstructural or even smaller length scales by transfering the observed mechanisms to the macro-scale in a suited manner. Currently efficient modelling tools have been developed for different length scales and there still exists a challenge in passing relevant information between models on different scales. This chapter aims at overviewing the current stage of modelling tools at different length scales, discussing the possible approaches to bridge different length scales, and reporting successful multiscale modelling applications.
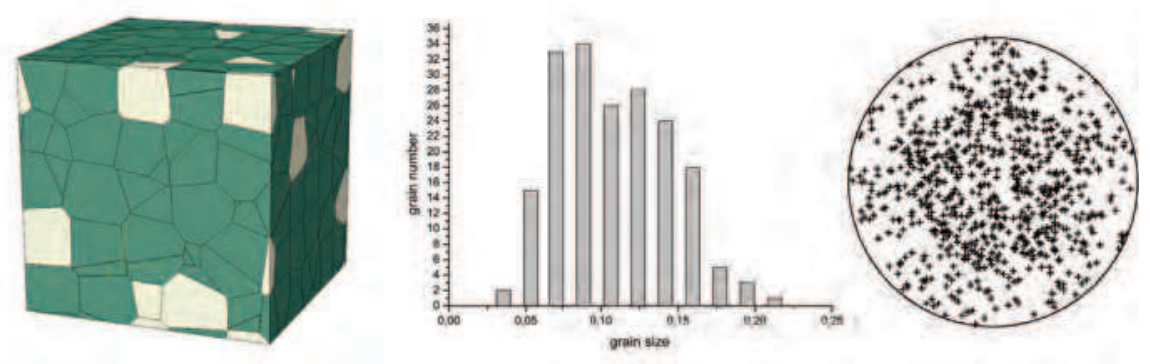

Fig. 1. Multiphase polycrystalline RVE (right) with $90 \%$ matrix and $10 \%$ precipitate. The grain size has a normal distribution (middle) and the [111] polfigure (left) shows a random texture. 


\section{Generating realistic material microstructures}

The current advanced high strength steels (AHSS) such as dual phase steels, transformation induced plasticity (TRIP) steels, twin induced plasticity (TWIP) steels and martensitic steels are all multiphase polycrystalline materials. In order to model the the macroscopic mechanical properties such as yield stress, work hardening rate and elongation to fracture, one has to build a representative volume element (RVE) for each macroscale material point and investigate the local deformation of each material point within the RVE, and then make a volume average. In this micro-macro-transition procedure, in order to reduce the computational costs the statistically similar representative volume elements (SSRVEs) have been developed to replace real microstructures from metallurgical images by Schröder et al. (2010).

Considering the real microstructure of multiphase materials, during the representative volume element generation one should consider grain shape distribution, crystalline orientation distribution, grain boundary misorientation angle distribution and volume fraction of different phases. Figure 1 is an example of the RVE we have generated for TRIP steels where the Voronoi tessellation algorithm has been used.

Recent studies (Lu et al., 2009; 2004) show bulk specimens comprising nanometer sized grains with embedded lamella with coherent, thermal and mechanical stable twin boundaries exhibiting high strength and considerable ductility at the same time. These materials have higher loading rate sensitivity, better tolerance to fatigue crack initiation, and greater resistance to deformation. Under this condition, the RVE with nanometer sized twin lamella inside nanometer sized twin lamella inside nanometer sized grains will help us to understand existing material behavior and design new materials.

Assume two orientations $\mathbf{Q}_{\mathrm{I}}$ and $\mathbf{Q}_{\mathrm{II}}$ have the twin relationship in $(1,1,1)$ habit plane along $[1,1,-2]$ twinning direction. For any vector $\mathbf{V}$, these two tensors will map as $\mathbf{v}_{I}=\mathbf{Q}_{I} \mathbf{V}$ and $\mathbf{v}_{\mathrm{II}}=\mathbf{Q}_{\mathrm{II}} \mathbf{V}$. The twin relationship between $\mathbf{v}_{\mathrm{I}}$ and $\mathbf{v}_{\mathrm{II}}$ is easier to see in the local twin coordinate system with $\mathbf{x}^{\prime} / /[1,1,-2]$ and $\mathbf{z}^{\prime} / /[1,1,1]$ rather than in the global coordinate system $[\mathbf{x}, \mathbf{y}, \mathbf{z}]$. We define a orthogonal tensor $\mathbf{R}_{\mathrm{L}}$ for the mapping from global coordinate system to the local coordinate system

$$
R_{\mathrm{L} i j}=\left[\begin{array}{ccc}
\frac{1}{\sqrt{6}} & \frac{1}{\sqrt{2}} & \frac{1}{\sqrt{3}} \\
\frac{1}{\sqrt{6}} & \frac{-1}{\sqrt{2}} & \frac{1}{\sqrt{3}} \\
\frac{-2}{\sqrt{6}} & 0 & \frac{1}{\sqrt{3}}
\end{array}\right]
$$

and another tensor $\mathbf{R}_{\mathrm{M}}$ for the mirror symmetry operation

$$
R_{\mathrm{M} i j}=\left[\begin{array}{rrr}
1 & 0 & 0 \\
0 & 1 & 0 \\
0 & 0 & -1
\end{array}\right]
$$

and get the twin relationship in the local twin coordinate system

$$
\left(\mathbf{R}_{\mathrm{L}} \mathbf{Q}_{\mathrm{I}} \mathbf{R}_{\mathrm{L}}^{\mathrm{T}}\right) \mathbf{v}^{\prime}=\mathbf{R}_{\mathrm{M}}\left(\mathbf{R}_{\mathrm{L}} \mathbf{Q}_{\mathrm{II}} \mathbf{R}_{\mathrm{L}}^{\mathrm{T}}\right) \mathbf{v}^{\prime}
$$


Because $\mathbf{v}^{\prime}$ is a arbitrary vector in the $\left[\mathbf{x}^{\prime}, \mathbf{y}^{\prime}, \mathbf{z}^{\prime}\right]$ coordinate system equation 3 will reduce to

$$
\mathbf{R}_{\mathrm{L}} \mathbf{Q}_{\mathrm{I}} \mathbf{R}_{\mathrm{L}}^{\mathrm{T}}=\mathbf{R}_{\mathrm{M}}\left(\mathbf{R}_{\mathrm{L}} \mathbf{Q}_{\mathrm{II}} \mathbf{R}_{\mathrm{L}}^{\mathrm{T}}\right)
$$

and we find the relationship between $\mathbf{Q}_{\mathrm{I}}$ and $\mathbf{Q}_{\mathrm{II}}$ as

$$
\mathbf{Q}_{\mathrm{II}}=\mathbf{R}_{\mathrm{L}}^{\mathrm{T}} \mathbf{R}_{\mathrm{M}}^{-1} \mathbf{R}_{\mathrm{L}} \mathbf{Q}_{\mathrm{I}} \text {. }
$$

The crystal orientation $\mathbf{Q}_{\mathrm{I}}$ has been assigned to the material point at the centre of the individual grain with coordinate $\left[\mathbf{x}_{0}^{\prime}, \mathbf{y}_{0}^{\prime}, \mathbf{z}_{0}^{\prime}\right]$. The orientation $\mathbf{Q}_{\text {II }}$ will be assigned to the twinned material point with coordinate $\left[\mathbf{x}_{1}^{\prime}, \mathbf{y}_{1}^{\prime}, \mathbf{z}_{1}^{\prime}\right]$ when the distance between this point and the grain center along the habit plane normal direction and the lamella thickness $d$ satisfy

$$
\left[(2 k-1)-\frac{1}{2}\right] \cdot d<\left|z_{1}^{\prime}-z_{0}^{\prime}\right| \leq\left[2 k-\frac{1}{2}\right] \cdot d \text { with } k=1,2,3, \ldots
$$

Otherwise orientation $\mathbf{Q}_{\mathrm{I}}$ will be assigned to this material point.

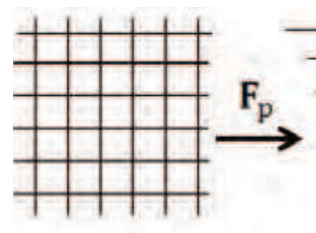

$X$

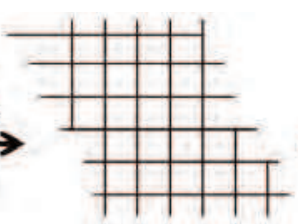

$\tilde{x}$

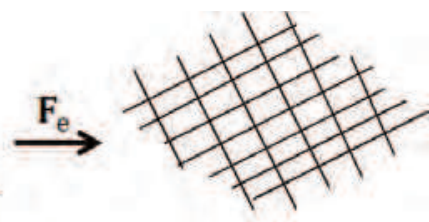

$x$

Fig. 2. The multiplicative decomposition of the deformation gradient where the plastic deformation is accommodated by dislocation slip.

\section{Constitutive models based on continuum mechanics}

\subsection{Kinematics}

For large strains the elastic and plastic deformation can be separated consistently. We follow the well-known multiplicative decomposition proposed by Lee (1969) (see Figure 2) of the deformation gradient tensor $\mathbf{F}$

$$
\mathbf{F}=\frac{\partial \mathbf{x}}{\partial \mathbf{X}}=\frac{\partial \mathbf{x}}{\partial \widetilde{\mathbf{x}}} \frac{\partial \widetilde{\mathbf{x}}}{\partial \mathbf{X}}=\mathbf{F}_{\mathrm{e}} \mathbf{F}_{\mathrm{p}}
$$

where $F_{e}$ is the elastic part comprising the stretch and rotation of the lattice, and $\mathbf{F}_{\mathrm{p}}$ corresponds to the plastic deformation. The lattice rotation $\mathbf{R}_{\mathrm{e}}$ and stretch $\mathbf{U}_{\mathrm{e}}$ are included in the mapping $\mathbf{F}_{\mathrm{e}}$. They can be calculated by the polar decomposition $\mathbf{F}_{\mathrm{e}}=\mathbf{R}_{\mathrm{e}} \mathbf{U}_{\mathrm{e}}$, i.e., the texture evolution is included in this part of the deformation. Furthermore, two rate equations can be derived for the elastic and the plastic deformation gradients as

$$
\begin{gathered}
\dot{\mathrm{F}}_{\mathrm{e}}=\mathbf{L F}_{\mathrm{e}}-\mathbf{F}_{\mathrm{e}} \mathbf{L}_{\mathrm{p}} \\
\dot{\mathrm{F}}_{\mathrm{p}}=\mathbf{L}_{\mathrm{p}} \mathrm{F}_{\mathrm{p}}
\end{gathered}
$$


where $\mathbf{L}=\dot{\mathbf{F F}}^{-1}$ and $\mathbf{L}_{\mathrm{p}}=\dot{\mathbf{F}}_{\mathrm{p}} \mathbf{F}_{\mathrm{p}}^{-1}$ are the total and the plastic velocity gradients defined in the current and the unloaded configuration respectively. Because the stress produced by the elastic deformation can supply driving forces for dislocation slip, twinning formation and phase transformation which can accommodate the plastic deformation, $\mathbf{F}_{\mathrm{e}}$ and $\mathbf{F}_{\mathrm{p}}$ are not independent. If the total deformation process is known, the elastic and plastic deformation evolutions can be determined through solving equations 7,8 and 9 .

When the eigen-deformation $\mathbf{F}_{\mathrm{t}}$ of phase transformation and the plastic deformation $\mathbf{F}_{\mathrm{p}}$ of dislocation slip coexist, the multiply decomposition (see Figure 3) should be reformulated as the following

$$
\mathbf{F}=\frac{\partial \mathbf{x}}{\partial \widetilde{\mathbf{x}}^{\prime}} \frac{\partial \widetilde{\mathbf{x}}^{\prime}}{\partial \widetilde{\mathbf{x}}} \frac{\partial \widetilde{\mathbf{x}}}{\partial \mathbf{X}}=\mathbf{F}_{\mathrm{e}} \mathbf{F}_{\mathrm{t}} \mathbf{F}_{\mathrm{p}}
$$

The evolution of $\mathbf{F}_{\mathbf{t}}$ is controlled by the transformed volume fraction $f_{\alpha}$ because the eigen-deformation $\widetilde{\mathbf{H}}_{\mathrm{t}}^{\alpha}$ of each transformation system with unit volume fraction is a constant tensor

$$
\begin{gathered}
\mathbf{F}_{\mathrm{t}}=\mathbf{I}+\sum_{\alpha=1}^{N_{T}} f^{\alpha} \widetilde{\mathbf{H}}_{\mathrm{t}}^{\alpha} \\
\dot{\mathbf{F}}_{\mathbf{t}}=\sum_{\alpha=1}^{N_{T}} \dot{f}^{\alpha} \widetilde{\mathbf{H}}_{\mathrm{t}}^{\alpha} .
\end{gathered}
$$

where $N_{T}$ is the total number of transformation system.

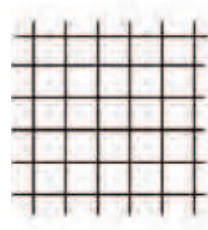

$X$
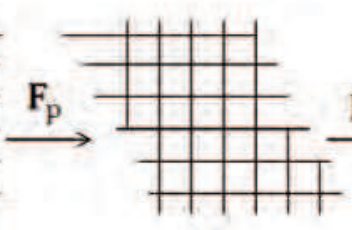

$\tilde{\boldsymbol{x}}$

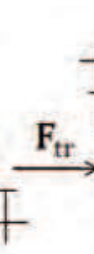

(1)

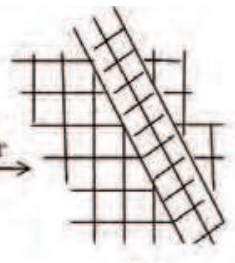

$\tilde{\boldsymbol{x}}^{\prime}$

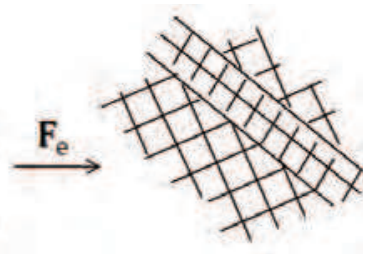

$x$

Fig. 3. The multiplicative decomposition of the deformation gradient when dislocation slip and phase transformation coexist.

\subsection{The elastic deformation}

For the dislocation slip case, the plastic deformation $\mathbf{F}_{\mathrm{p}}$ will not change the lattice orientation, i.e., we can use a constant stiffness tensor $\widetilde{\mathbb{K}}_{0}$ for the stress calculations and define the elastic law in the unloaded configuration. By defining the second Piola-Kirchhoff stress tensor $\widetilde{\mathbf{S}}$ in the unloaded configuration and its work conjugated elastic Green strain tensor $\tilde{\mathbf{E}}$, the elastic law is derived as

$$
\widetilde{\mathbf{S}}=\widetilde{\mathbb{K}}_{0} \widetilde{\mathbf{E}}
$$

with

$$
\widetilde{\mathbf{E}}=\frac{1}{2}\left(\mathrm{~F}_{\mathrm{e}}^{\mathrm{T}} \mathbf{F}_{\mathrm{e}}-\mathbf{I}\right)
$$

where $\mathbf{I}$ is the second order unity tensor. When the variational principle of the FEM is formulated in the reference or current configuration, the second Piola-Kirchhoff stress $\mathbf{S}$ or 
the Cauchy stress $\sigma$ amount to

$$
\begin{aligned}
\mathbf{S} & =\mathbf{F}_{\mathrm{p}}^{-1} \widetilde{\mathbf{S}} \mathbf{F}_{\mathrm{p}}^{-T} \\
\boldsymbol{\sigma} & =\frac{1}{J} \mathbf{F}_{\mathrm{e}} \widetilde{\mathbf{S}} \mathbf{F}_{\mathrm{e}}^{T}
\end{aligned}
$$

where $J=\operatorname{det}(\mathbf{F})=\operatorname{det}\left(\mathbf{F}_{\mathrm{e}}\right)$, which means the isochoric plastic deformation is assumed, i.e., the volume change is always purely elastic.

In a polycrystal the different grains have different initial orientations. Therefore, in the global coordinate system, different stiffness tensors, slip plane normals and slip directions should be specified for every crystal. In order to use only one set of data, a virtual deformation step is introduced before the calculation in the following form: $\mathbf{F}_{\mathrm{p} 0}$ is set as the initial value for $\mathbf{F}_{\mathrm{p}}$. By choosing $\mathrm{F}_{\mathrm{e} 0}$ to satisfy

$$
\mathbf{F}_{\mathrm{e} 0} \mathbf{F}_{\mathrm{p} 0}=\mathbf{I}, \quad \mathbf{F}_{\mathrm{e} 0}, \mathbf{F}_{\mathrm{p} 0} \in \text { Orth. }
$$

the starting value for $\mathbf{F}$ amounts to $\mathbf{I}$ as desired. If one adopts the Bunge Euler angle $\left(\varphi_{1}, \Phi, \varphi_{2}\right)$ to define the crystal orientation, the matrix of the elastic deformation gradient amounts to

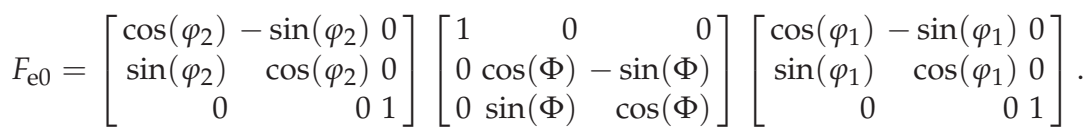

\subsection{The dislocation slip based plastic deformation}

The plastic deformation mechanism discussed here is the slip mechanism where dislocations slip in certain crystallographic planes along certain crystallographic directions to accommodate shape changes of the crystal. This is the most common mechanism in conventional metal forming processes.

The concept for describing displacement fields around dislocations in crystals was developed mathematically by Volterra and used for calculating elastic deformation fields by Orowan and Taylor in 1934 (Hirth \& Lothe, 1992). Dislocations are one dimensional lattice defects which can not begin or end inside a crystal, but must intersect a free surface, form a closed loop or make junctions with other dislocations. Due to energetic reasons there is a strong tendency for dislocations to assume a minimum Burgers vector, and to slide in the planes with maximum interplanar separation and along the most densely packed directions. Under the applied stress, the lattice deforms elastically, until stretched bonds near the dislocation core break and new bonds form. The dislocation moves step by step by one Burgers vector. It is the dislocation slip mechanism that can explain why the actually observed strength of most crystalline materials is between one to four orders of magnitude smaller than the intrinsic or theoretical strength required for breaking the atom bonds without the presence of dislocations.

In order to set the connection between the continuum variables and the process of dislocation slip, we have to determine the shear amount of individual slip systems. The slip systems are mathematically described by the Schmid tensor $\widetilde{\mathbf{M}}^{\alpha}=\widetilde{\mathbf{d}}^{\alpha} \otimes \widetilde{\mathbf{n}}^{\alpha}$ where $\widetilde{\mathbf{d}}^{\alpha}=\mathbf{b} / b$ expresses the slip direction, which is parallel to the Burgers vector $\mathbf{b}$, but normalised, and $\widetilde{\mathbf{n}}^{\alpha}$, the slip plane normal with respect to the undistorted configuration. Through calculating the line vector $\widetilde{\mathbf{l}}^{\alpha}=\widetilde{\mathbf{d}}^{\alpha} \times \widetilde{\mathbf{n}}^{\alpha}$ we can define one local coordinate system for slip system $\alpha$ as $\left[\widetilde{\mathbf{1}}^{\alpha}, \widetilde{\mathbf{d}}^{\alpha}, \widetilde{\mathbf{n}}^{\alpha}\right]$, which is useful in the later GND calculations. 
For the FCC crystal structure, the close-packed planes $\{111\}$ and close-packed directions $\langle 110\rangle$ form 12 slip systems. For the BCC crystal structure, the pencil glide phenomenon is observed, which resembles slip in a fixed direction on apparently random planes. In literature, experimental studies have shown that for BCC crystals the slip direction is along $\langle 111\rangle$, and three groups of slip planes exist, including $\{110\},\{112\}$ and the less common $\{123\}$ planes. Totally there are 48 slip systems for BCC crystals. Therefore, for FCC and BCC crystals it is possible to supply five independent slip systems to accommodate any arbitrary external plastic deformation, and in the middle temperature range the slip is the main mechanism for plastic deformations. For the HCP crystal, the slip system number is dependent on the axis ratio of the HCP unit cell. When this ratio assumes values in a certain range as discussed by Gottstein (2004), only two independent slip systems exist, and it is impossible to accommodate a arbitrary deformation by slip steps. As a result, mechanical twins appear during plastic deformation.

Among all of the dislocations in one slip system, only the mobile dislocations can produce plastic deformation, and their speed can be determined by the forces acting on them. In general, the driving force is related to external loads, short range isotropic resistance of dislocation interactions and long range back stress of dislocation pile-ups and lattice frictions. The widely-adopted constitutive assumption for crystal plasticity reads

$$
\mathbf{L}_{\mathrm{p}}=\sum_{\alpha=1}^{N_{S}} \dot{\gamma}^{\alpha} \widetilde{\mathbf{M}}^{\alpha}, \quad \dot{\mathbf{F}}_{\mathrm{p}}=\sum_{\alpha=1}^{N_{S}} \dot{\gamma}^{\alpha} \widetilde{\mathbf{M}}^{\alpha} \mathbf{F}_{\mathrm{p}}
$$

where $\dot{\gamma}^{\alpha}$ is the slip rate on slip system $\alpha$ within the intermediate configuration $\widetilde{\mathbf{x}}$, and $N_{S}$, the total number of slip systems.

When a part of material is transferred into another lattice with volume fraction $f=\sum_{\alpha=1}^{N_{T}} f^{\alpha}$ which can only deform elastically, equation 18 has been modified as

$$
\mathbf{L}_{\mathrm{p}}=(1-f) \sum_{\alpha=1}^{N_{S}} \dot{\gamma}^{\alpha} \widetilde{\mathbf{M}}^{\alpha}, \quad \dot{\mathbf{F}}_{\mathrm{p}}=(1-f) \sum_{\alpha=1}^{N_{S}} \dot{\gamma}^{\alpha} \widetilde{\mathbf{M}}^{\alpha} \mathbf{F}_{\mathrm{p}}
$$

\subsubsection{The Orowan equation}

Commonly used expressions for the relation of the shear rate, $\dot{\gamma}$, and the resolved shear stress, $\tau$, include a phenomenological viscoplastic law in the form of a power law by Peirce et al. (1982), and more physically-based ones such as those of Kocks et al. (1975) and Nemat-Nasser et al. (1998), which can take account of rate and temperature dependencies. In this paper we use the Orowan equation to calculate the plastic shear rate $\dot{\gamma}$ of each slip system as a function of the mobile dislocation density, $\rho_{\mathrm{m}}$, on that slip system

$$
\dot{\gamma}=\rho_{\mathrm{m}} b v
$$

where the average velocity of the mobile dislocations, $v$, is a function of the resolved shear stress, $\tau$, of the dislocation densities, $\rho_{\mathrm{M}}, \rho_{\mathrm{SSD}}$ and $\rho_{\mathrm{GND}}$ and its gradient, the average GND 
pile-up size, $L$, and of the temperature, $\theta$; i.e.,

$$
v=v\left(\tau, \rho_{\mathrm{SSD}}, \rho_{\mathrm{GND}}, \frac{\partial \rho_{\mathrm{GND}}}{\partial X}, L, \theta\right)
$$

The resolved shear stress, $\tau$, is the projection of the stress measure onto the slip system. In the case of infinitesimally small elastic stretches $\|\widetilde{\mathbf{C}}\|=\left\|\widetilde{\mathbf{F}}_{\mathrm{e}}^{\mathrm{T}} \widetilde{\mathbf{F}}_{\mathrm{e}}\right\|<<1$, the resolved shear stress, $\tau$, within the intermediate configuration $\widetilde{\mathbf{x}}$ can be approximated by following Kalidindi et al. (1992)

$$
\tau^{\alpha}=\widetilde{\mathbf{S}} \widetilde{\mathbf{C}} \cdot \widetilde{\mathbf{M}}^{\alpha} \cong \tilde{\mathbf{S}} \cdot \widetilde{\mathbf{M}}^{\alpha}
$$

In order to accommodate a part of the external plastic deformation, the mobile dislocations, $\rho_{\mathrm{M}}$, must overcome the stress field of the parallel dislocations, $\rho_{\mathrm{P}}$, which cause the passing stress. They must also cut the forest dislocations, $\rho_{\mathrm{F}}$, with the aid of thermal activation. We define the parallel dislocation density and the forest dislocation density as: $\rho_{\mathrm{P}}$ for all dislocations parallel to the slip plane, and $\rho_{\mathrm{F}}$ for the dislocations perpendicular to the slip plane. Both $\rho_{\mathrm{SSD}}$ and $\rho_{\mathrm{GND}}$ are contributing to $\rho_{\mathrm{F}}$ and $\rho_{\mathrm{P}}$

$$
\rho_{\mathrm{F}}^{\alpha}=\sum_{\beta=1}^{N_{S}} \chi^{\alpha \beta}\left(\rho_{\mathrm{SSD}}^{\beta}+\rho_{\mathrm{GND}}^{\beta}\right)\left|\cos \left(\widetilde{\mathbf{n}}^{\alpha}, \widetilde{\mathbf{t}}^{\beta}\right)\right|
$$

and

$$
\rho_{\mathrm{P}}^{\alpha}=\sum_{\beta=1}^{N_{S}} \chi^{\alpha \beta}\left(\rho_{\mathrm{SSD}}^{\beta}+\rho_{\mathrm{GND}}^{\beta}\right)\left|\sin \left(\widetilde{\mathbf{n}}^{\alpha}, \widetilde{\mathbf{t}}^{\beta}\right)\right|
$$

where we introduce the interaction strength, $\chi^{\alpha \beta}$, between different slip systems, which includes the self interaction strength, coplanar interaction strength, cross slip strength, glissile junction strength, Hirth lock strength, and Lomer-Cottrell lock strength. One can go further to see the definition of these interactions in literature (Devincre et al., 2008; Madec et al., 2008). In this formulation we only consider edge dislocations owing to their limited mobility for the FCC crystal, and use a single set of interaction strengths for both SSDs and GNDs.

With the help of the forest dislocation density $\rho_{\mathrm{F}}$, we can determine the average jump distance of the mobile dislocation and the activation volume for the thermal activated forest dislocation cutting event

$$
\lambda=\frac{c_{1}}{\sqrt{\rho_{F}}}
$$

and

$$
V=c_{2} b^{2} \lambda
$$

where $c_{1}$ and $c_{2}$ are constants to reflect the real dislocation line configuration which is more complicated than the schematic pictures we use here.

With the help of the parallel dislocation density $\rho_{\mathrm{P}}$ and the gradient of GND density $\frac{\partial \rho_{\mathrm{GND}}}{\partial \mathrm{X}}$, we can determine the average athermal passing stress $\tau_{\mathrm{p}}$ and back stress $\tau_{\mathrm{b}}$ as following

$$
\tau_{\mathrm{p}}=c_{3} G b \sqrt{\rho_{P}}
$$


and

$$
\tau_{\mathrm{b}}=G b L^{2} \frac{\partial \rho_{\mathrm{GND}}}{\partial X}
$$

where $c_{3}$ is the constant for the Taylor hardening mechanism. For reasons of simplicity, in equation 28 the back stress of one slip system only comes from the GND pile-up of this slip system. This equation can be easily extended to consider the back stress from all of the slip system at the same time.

Compared with flow rules in the literature which contain a constant reference shear rate and a constant rate sensitivity exponent, here a flow rule is derived based on the dislocation slip mechanism

$$
\dot{\gamma}=\left\{\begin{array}{cl}
\rho_{\mathrm{m}} b \lambda v_{0} \exp \left(-\frac{Q_{\text {slip }}}{\mathrm{k}_{\mathrm{B}} \theta}\right) \exp \left(\frac{\left|\tau+\tau_{\mathrm{b}}\right|-\tau_{\mathrm{p}}}{\mathrm{k}_{\mathrm{B}} \theta} V\right) \operatorname{sign}\left(\tau+\tau_{\mathrm{b}}\right) & \left|\tau+\tau_{\mathrm{b}}\right|>\tau_{\mathrm{p}} \\
0 & \left|\tau+\tau_{\mathrm{b}}\right| \leq \tau_{\mathrm{p}}
\end{array}\right.
$$

where $\mathrm{k}_{\mathrm{B}}$ is the Boltzmann constant, $v_{0}$ the attempt frequency and $Q_{\text {slip }}$ the effective activation energy.

Inside the flow rule given by equation 29 determination of the mobile dislocation density is a hard task. In some research work, the mobile dislocation density was found to be a small fraction of total dislocation density and is even treated as a constant. The more sophisticated model to deal with this dislocation density based on energy minimization can be found in Ma \& Roters (2004). For reasons of simplicity here the mobile dislocation density is treated as a constant number.

\subsubsection{Evolution of the dislocation densities}

There are four processes contributing to the evolution of the SSD density as discussed by Ma (2006). The lock forming mechanism between mobile dislocations and forest dislocations, the dipole forming mechanism between mobile dislocations with parallel line vectors, and anti-parallel Burgers vector determine the multiplication terms, while the annihilation term includes annihilation between one mobile dislocation with another immobile one and annihilation between two immobile dislocations. The often used Kocks-Mecking model, as discussed in Roters (1999), only adopts the locks formation and mobile-immobile annihilation mechanisms for the SSD evolution

$$
\dot{\rho}_{\mathrm{SSD}}=\left(c_{4} \sqrt{\rho_{\mathrm{F}}}-c_{5} \rho_{\mathrm{SSD}}\right) \dot{\gamma}
$$

Where $c_{4}$ and $c_{5}$ are constants used to adjust the locks and annihilation radius.

When plastic deformation gradients are present in a volume portion, GNDs must be introduced to preserve the continuity of the crystal lattice. A relation between a possible GND measure and the plastic deformation gradient has been proposed by Nye (1953). This approach has been later extended to a more physically motivated continuum approach to generally account for strain gradient effects by Dai \& Parks (1997). Following these pioneering approaches, we use as a dislocation density tensor, $\Lambda$, for a selected volume portion to 
calculate the net Burgers vector for an area

$$
\Lambda=\overline{\mathbf{b}} \otimes \overline{\mathbf{l}}=-\left(\nabla_{\mathrm{X}} \times \mathbf{F}_{\mathrm{p}}\right)^{\mathrm{T}}
$$

where $\nabla_{X}=\partial / \partial \mathbf{X}$, is defined as the derivative with respect to the reference coordinates and $\overline{\mathbf{b}}$ and $\overline{\mathbf{l}}$ are, respectively, the net Burgers vector and net line vector after an volume average operation. Using equation (31) the resulting Burgers vector for a circuit with an arbitrary orientation can be calculated. In general this tensor is non-symmetric and it can be mapped to nine independent slip systems in a unique fashion. For the FCC crystal structure with its 12 slip systems, only 5 systems are independent according to the von Mises-Taylor constraint. This implies that it is impossible to calculate the exact amount of GNDs for every slip system in a unique way. Nevertheless, we can project $\Lambda$ to each of the slip systems to determine the Burgers vector of the edge and screw type GNDs for the pass stress and backing stress calculation

$$
\mathbf{b}_{\mathrm{GNDe}}^{\alpha}=\left(\widetilde{\mathbf{d}}^{\alpha} \cdot \boldsymbol{\Lambda} \cdot \widetilde{\mathbf{l}}^{\alpha}\right) \widetilde{\mathbf{d}}^{\alpha}
$$

and

$$
\mathbf{b}_{\mathrm{GNDs}}^{\alpha}=\left(\widetilde{\mathbf{d}}^{\alpha} \cdot \boldsymbol{\Lambda} \cdot \widetilde{\mathbf{d}}^{\alpha}\right) \widetilde{\mathbf{d}}^{\alpha} .
$$

Furthermore we also can calculate the GND density as the following

$$
\rho_{\mathrm{GND}}^{\alpha}=\left(\left\|\mathbf{b}_{\mathrm{GNDe}}^{\alpha}\right\|+\left|\mathbf{b}_{\mathrm{GNDs}}^{\alpha}\right|\right) / b .
$$



Fig. 4. A transformation system of the austenite-martensite phase transformation.

\subsection{Eigenstrain of phase transformations}

The transformation-induced plasticity (TRIP) assisted steels are mixtures of allotriomorphic ferrite, bainite and retained austenite. Experimental and modelling publications have highlighted that the transformation of retained austenite to martensite under the influence of a applied stress or strain can improve material ductility and strength efficiently, as shown by Bhadeshia (2002).

According to the geometrically nonlinear theory of martensitic transformations (Bhattacharya, 1993; Hane \& Shield, 1998; 1999) there are 24 transformation systems and they are constructed by two body-centered tetragonal (BCT) variants with relative rotations and volume fractions, in order to produce habit planes between austenite and martensite arrays and pairwise arranging twin related variant lamellas. Each transformation system corresponds to one 
constant shape strain vector, $\widetilde{\mathbf{v}}_{s}^{\alpha}$, and one constant habit plane normal vector, $\widetilde{\mathbf{v}}_{n}^{\alpha}$, see Figure 4 . Following the classical Kurdjumov-Kaminsky relations, these two vectors are influenced by the carbon concentration through the lattice parameter magnitude variation (Hane \& Shield, 1998; Wechsler et al., 1953). The eigenstrain of the transformation system $\alpha$ amounts to

$$
\widetilde{\mathbf{H}}_{\mathrm{t}}^{\alpha}=\widetilde{\mathbf{v}}_{s}^{\alpha} \otimes \widetilde{\mathbf{v}}_{n}^{\alpha}
$$

As an example in the literature (Kouznetsova \& Geers, 2008; Tjahjanto, 2008), the shape strain vector and the habit plane normal vector with respect to specific carbon concentrations have been determined and listed. Because the shape strain vector and the habit plane normal vector are explicit functions of austensite and martensite lattice parameters, the components of the two vectors are irrational. $\widetilde{\mathbf{H}}_{\mathfrak{t}}^{\alpha}$ is similar to the Schmid tensor $\widetilde{\mathbf{M}}^{\alpha}$ of dislocation slip except that there is volume change $\operatorname{det}\left(\widetilde{\mathbf{H}}_{\mathrm{t}}^{\alpha}\right)>1$.

In literature, the austenite-martensite phase transformation has been formulated as stress and strain driving mechanisms among different temperature regions. The stress controlled transformation often occurs at lower temperatures where the chemical driving force is so high that a external load below austenite yield stress can help the already existing martensite nuclei to grow. At strain controlled transformation regions at higher temperatures, the chemical driving force is so low that additional loads which are higher than the yield stress are needed in order for existing nuclei to continue grow. Due to the fact that plastic deformation in austenite is easier than spontaneous martensite formation, the transformation has to be continued by new nuclei formation at the shear band intersection region according to Olson \& Cohen (1972).

Following the Olson-Cohen model, the transformation kinetics are formulated in meso-scale on the micro-band level. At first the shear band density is estimated, then the intersection frequency of shear bands is calculated and lastly the nucleation producing probability is evaluated. This governing equation for martensite volume fraction reads

$$
\dot{f}^{\alpha}=\left\{\begin{array}{cl}
c_{6}\left(1-\sum_{\beta=1}^{N_{\mathrm{T}}} f_{\beta}\right)\left|\tau_{\alpha}\right|^{c_{7}} \sum_{i, j=1}^{N_{\mathrm{S}}}\left(1-\left|\widetilde{\mathbf{l}}_{i} \cdot \widetilde{\mathbf{l}}_{j}\right|\right)\left(1-\left|\widetilde{\mathbf{n}}_{i} \cdot \widetilde{\mathbf{n}}_{j}\right|\right) \sqrt{\left|\dot{\gamma}_{i} \dot{\gamma}_{j}\right|} & \tau_{\alpha}>0 \\
0 & \tau_{\alpha} \leq 0
\end{array}\right.
$$

where $N_{\mathrm{T}}$ and $N_{\mathrm{S}}$ are the total number of transformation systems and slip systems with, respectively, $c_{6}$ and $c_{7}$ as two fitting parameters to control the transformation kinetics; and

$$
\tau^{\alpha} \cong \tilde{\mathbf{S}} \cdot \widetilde{\mathbf{H}}_{\mathrm{t}}^{\alpha}
$$

the resolved stress in the transformation system $\alpha$ with includes the shear part and the tensile or compression part at the same time. Indeed, in equation 36 the phase transformation is controlled by the external load potential minimisation.

Because the transformed martensitic phase includes twinned wedge microstructures, the Frank-Read dislocation source may suffer higher resistance compared with the original austenitic phase. The dislocation slip based plasticity of martensite has been neglected here. 

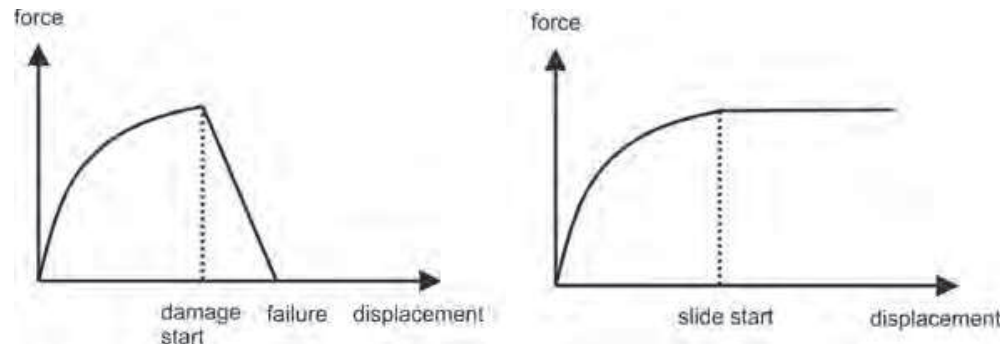

Fig. 5. Schematic drawings of cohesive behaviour of grain boundaries along normal (left) and tangential (right) directions.

\subsection{Cohesive zone model for grain and phase boundaries}

In experimental works and atomistic simulations with respect to deformation of nanocrystalline materials, dislocation glide inside the grains and grain boundary sliding have both been reported. It is obvious that grain-boundary sliding and separation mechanisms begin to play important roles in the overall inelastic response of a polycrystalline material when the grain-size decreases and dislocation activity within the grain interior becomes more difficult. In recent work, the atomic bonds across grain boundaries have been characterized with ab initio calculations within the framework of the density functional theory (Janisch et al., 2010). In this work not only the energetics of grain boundaries have been characterized, but also the mechanical response of a grain boundary to applied loads is studied. Such information can be used to parameterize cohesive zone models based on ab initio calculations.

The cohesive zone model is useful for RVE models of polycrystals, in situations when grain boundary deformation needs to be taken into account explicitely, e.g. when grain boundary sliding or damage initiation at grain boundaries or phase bounaries has to be considered. By adjusting the cohesive zone parameters for grain boundary sliding and opening the competing mechanisms of bulk material deformation and grain boundary accommodated deformation can be studied. Furthermore, it is also possible to investigate damage nucleation at GB triple junctions.

We follow Wei \& Anand (2004) to generate a rate independent cohesive zone (CZ) modelling approach for the reasons of simplicity. The velocity jump across a cohesive surface has been additively decomposed into a elastic and a plastic part as follow

$$
\dot{\mathbf{u}}=\dot{\mathbf{u}}_{\mathrm{e}}+\dot{\mathbf{u}}_{\mathrm{p}}
$$

The elastic relative velocities are connected with its power-conjugate traction rate by the interface elastic stiffness tensor

$$
\dot{\mathbf{t}}=\mathbf{K} \dot{\mathbf{u}}_{\mathrm{e}}=\mathbf{K}\left(\dot{\mathbf{u}}-\dot{\mathbf{u}}_{\mathrm{p}}\right)
$$

For some special grain boundaries there may exist glide anisotropy inside the grain boundary plane, although our knowledge about this topic is far away from formulating this anisotropy for general grain boundaries. So, we have to assume isotropic plastic deformation property inside the grain boundary, and the trace vector, displacement vector and resistance vector are 
defined in the local coordinate $\left[\mathbf{t}_{I}, \mathbf{t}_{I I}, \mathbf{n}\right]$, where $\mathbf{n}$ is aligned with the normal to the interface, $\mathbf{t}_{I}$ and $\mathbf{t}_{I I}$ in the tangent plane at the point of the interface under consideration.

According to the rate independent assumption for the loading condition, the hardening rate $\dot{\mathbf{s}}$ has to be fast enough to balance the load $\dot{t}$

$$
\dot{\mathbf{t}}=\dot{\mathbf{s}}
$$

or

$$
\mathbf{K}\left(\dot{\mathbf{u}}-\dot{\mathbf{u}}_{\mathrm{p}}\right)=\mathbf{H} \dot{\mathbf{u}}_{\mathrm{p}}
$$

where $\mathbf{H}$ is the hardening matrix and its components are state variables of the cohesive zone model. The evolution law of the hardening matrix

$$
\dot{\mathbf{H}}=\dot{\mathbf{H}}\left(\mathbf{H}, \dot{\mathbf{u}}_{\mathrm{p}}\right)
$$

can be obtained by experimental date fitting or can directly come from the molecular dynamic and ab-initial calculations. Here we adopted the phenomenological hardening rule proposed by Wei \& Anand (2004) for the cohesive zone model along the normal direction. For the cohesive zone model along the tangential direction, the failure displacement has been set to infinite to consider the grain boundary glide phenomenon, see Figure 5.

\section{Numerical approaches}

Starting from the stress equilibrium state, for a given time step and velocity gradient one has to calculate the new stress state, while at the same time considering the evolution of state variables including plastic shear amount $\gamma$ determined by equation 29 , statistically stored dislocation density $\rho_{\text {SSD }}$ determined by equation 30 and geometrically necessary dislocation density $\rho_{\mathrm{GND}}$ determined by equation 34 for each slip system, the transformed volume fraction $f$ determined by equation 36 for each transformation system and the hardening matrix $\mathbf{H}$ determined by equation 42 for the cohesive zone model of grain boundaries.

\subsection{Finite element method}

Based on the Abaqus platform (ABAQUS, 2009), we have developed the user material subroutines UMAT for the bulk material and UINTER for the grain boundary to solve the stress equilibrium and state variable evolution problems. In this approach, except for the plastic strain gradient used for the geometrically necessary dislocation density calculation, which is adopted from last converged time point, all of state variables are calculated by an implicit method.

\subsection{Discrete fast Fourier transformation method}

If the representative volume element has a very complicated microstructure and obeys a periodic boundary condition, the stress equilibrium and state variable evolution problems can be solved by the discrete fast Fourier transformation (FFT) method proposed in the literature (Lebensohn, 2001; Michel et al., 2000; 2001). 
According to this approach the material points of the real RVE are approximated to inclusions inside one homogeneous matrix, the property of which can be determined as volume average of these inclusions. After the regular discretisation of the RVE, in the current configuration the inclusion at location $\mathbf{x}^{\prime}$ has stress increment $\Delta \sigma^{I}$, strain increment $\Delta \boldsymbol{\epsilon}^{I}$ and stiffness $\mathbb{C}^{I}=$ $\partial \Delta \sigma^{I} / \partial \Delta \epsilon^{I}$, and the matrix material point at the same location has stress increment $\Delta \sigma^{M}$, strain increment $\Delta \epsilon^{M}$ and stiffness $\overline{\mathbb{C}}$.

Assuming we deal with a deformation control process, at the beginning of the iteration loop

$$
\Delta \epsilon^{I}=\Delta \epsilon^{M}=\overline{\Delta \epsilon}
$$

where $\overline{\Delta \epsilon}$ is the given fixed strain increment. Because each material point has the same volume and shape, the matrix stiffness can be determined simply as

$$
\overline{\mathbb{C}}=\sum_{\mathbf{x}^{\prime} \in R V E} \frac{1}{N} \mathbb{C}^{I}
$$

where $N$ is the total number of inclusions. At this stage, for the inclusions the strain field satisfies deformation compatibility while the stress field does't satisfy the stress equilibrium.

\subsubsection{Stress and strain increment of matrix material points}

The polarized stress increment field $\Delta \sigma^{I}-\Delta \sigma^{M}$ can cause a strain increment field in the matrix. Because the matrix material is homogeneous and suffering a periodic boundary condition, this strain increment can be calculated efficiently with help of Green's function and discrete Fourier transformation. With the help of the delta function

$$
\delta\left(\mathbf{x}-\mathbf{x}^{\prime}\right)= \begin{cases}1 & \mathbf{x}=\mathbf{x}^{\prime} \\ 0 & \mathbf{x} \neq \mathbf{x}^{\prime}\end{cases}
$$

a unit force $\delta_{m i}\left(\mathbf{x}-\mathbf{x}^{\prime}\right)$ in $m$ plane along $i$ direction applying at $\mathbf{x}^{\prime}$ will cause a displacement field $G_{k m}\left(\mathbf{x}-\mathbf{x}^{\prime}\right)$ at $\mathbf{x}$ satisfying the stress equilibrium

$$
\overline{\mathrm{C}}_{i j k l} G_{k m, l j}+\delta_{m i}=0 .
$$

In order to solve equation 46 we have to transfer it into the frequency space

$$
-\overline{\mathbb{C}}_{i j k l} \hat{G}_{k m} \xi_{l} \xi_{j}+\delta_{m i}=0
$$

where $\xi$ represents the frequency. Through defining a second order tensor $A_{i k}=\overline{\mathbb{C}}_{i j k l} \xi_{l} \xi_{j}$ we find the displacement $\hat{G}_{k m}$ in the frequency space

$$
A_{i k} \hat{G}_{k m}=\delta_{i m} \quad \text { or } \quad \hat{\mathbf{G}}=\mathbf{A}^{-1} .
$$

When one transfers back $\hat{G}$ from the frequency space to the real physical space one can get the solution $\mathbf{G}$ of equation 46 . However, this is not necessary because we will solve the displacement field caused by the polarised stress in the frequency space. 
Based on the solution of equation 46, the displacement $\Delta \mathbf{u}^{M}$ with respect to the polarised stress field $\Delta \boldsymbol{\sigma}^{I}-\Delta \boldsymbol{\sigma}^{M}$ can be calculated with the help of Gauss's Theorem and the periodic arrangement of the RVE

$$
\Delta u_{k}^{M}=\sum_{\mathbf{x}^{\prime} \in R V E} G_{k m, n}\left(\Delta \sigma_{m n}^{I}-\Delta \sigma_{m n}^{M}\right) \Omega
$$

where $\Omega$ is the volume of the regular element inside the RVE. Furthermore we can calculate the gradient of the displacement

$$
\Delta u_{k, o}^{M}=\sum_{\mathbf{x}^{\prime} \in R V E} G_{k m, n o}\left(\Delta \sigma_{m n}^{I}-\Delta \sigma_{m n}^{M}\right) \Omega
$$

and transfer equation 50 into the frequency space, with the help of the convolution theorem:

$$
\Delta \hat{u}_{k, o}^{M}=-\sum_{\mathbf{x}^{\prime} \in R V E} \hat{G}_{k m} \xi_{n} \xi_{o}\left(\Delta \hat{\sigma}_{m n}^{I}-\Delta \hat{\sigma}_{m n}^{M}\right) \Omega .
$$

Through taking equation 48 into equation 51 we can calculate the displacement gradient in the frequency space

$$
\begin{aligned}
\Delta \hat{u}_{k, o}^{M} & =-\sum_{\mathbf{x}^{\prime} \in R V E} A_{k m}^{-1} \xi_{n} \xi_{o}\left(\Delta \hat{\sigma}_{m n}^{I}-\Delta \hat{\sigma}_{m n}^{M}\right) \Omega \\
& =-\sum_{\mathbf{x}^{\prime} \in R V E} \hat{\mathrm{S}}_{k o m n}\left(\Delta \hat{\sigma}_{m n}^{I}-\Delta \hat{\sigma}_{m n}^{M}\right) \Omega .
\end{aligned}
$$

where $\hat{\mathrm{S}}_{k o m n}$ is the compliance tensor in the frequency space for the material point at the real space $\mathbf{x}^{\prime}$. Finally, after one transfers equation 52 from frequency space back to the real physical space one gets the stress and strain increments for the matrix material points

$$
\begin{gathered}
\Delta \epsilon_{i j}^{M}=\frac{1}{2}\left(\Delta u_{i, j}^{M}+\Delta u_{j, i}^{M}\right) \\
\Delta \sigma_{i j}^{M}=\overline{\mathbb{C}}_{i j k l}^{-1} \Delta \epsilon_{k l}^{M} .
\end{gathered}
$$

\subsubsection{Stress and strain increment of inclusions}

For each material point at $\mathbf{x}$ when there is a strain misfit between matrix and inclusion $\Delta \epsilon^{I} \neq$ $\Delta \epsilon^{M}$ there will be a internal misfit stress $\bar{C}\left(\Delta \epsilon^{I}-\Delta \epsilon^{M}\right)$. The total strain energy increment amounts to

$$
\Delta E=\sum_{\mathbf{x}^{\prime} \in R V E}\left[\left(\boldsymbol{\sigma}^{I}+\Delta \boldsymbol{\sigma}^{I}\right) \cdot \Delta \boldsymbol{\epsilon}^{I}+\overline{\mathbb{C}}\left(\Delta \boldsymbol{\epsilon}^{I}-\Delta \boldsymbol{\epsilon}^{M}\right) \cdot\left(\Delta \boldsymbol{\epsilon}^{I}-\Delta \boldsymbol{\epsilon}^{M}\right) \Omega\right] .
$$

Now the procedure of finding stress equilibrium can be replaced with the procedure of achieving the total strain energy increment minimisation

$$
\nabla \cdot \sigma^{I}=\mathbf{0} \Longleftrightarrow \frac{\partial \Delta E}{\partial \Delta \boldsymbol{\epsilon}^{I}}=0 .
$$


Because each inclusion only has interaction with the matrix, for a fixed matrix property, material points at $\mathbf{x}$ and at $\mathbf{x}^{\prime}$ are independent if $\mathbf{x} \neq \mathbf{x}^{\prime}$. Under this condition, for each material point we can calculate the local strain increment which can keep the total strain energy increment minimisation

$$
\Delta \epsilon^{I}=\left(\mathbb{C}^{I}+\overline{\mathbb{C}}\right)^{-1}\left(\overline{\mathbb{C}} \Delta \epsilon^{M}-\sigma^{I}-\Delta \sigma^{I}\right)
$$

and with help of equation 57 , the stress increment $\Delta \sigma$ of inclusion at $\mathbf{x}^{\prime}$ can be easily recalculated by the constitutive law.

\subsubsection{Deformation compatibility}

In Lebensohn (2001) the deformation compatibility problem and the energy minimisation problems are joined together through adopting the Lagrange multiplier method. In this work, after the stress and strain calculation for inclusions we simply set the matrix material point deformation increment as

$$
\Delta \epsilon^{M}=\Delta \epsilon^{I} .
$$

The final stress and strain solution for inclusions satisfying stress equilibrium and strain compatibility will not be achieved until

$$
\sum_{\mathbf{x}^{\prime} \in R V E} \frac{1}{N}\left\|\Delta \boldsymbol{\epsilon}_{k}^{I}-\Delta \boldsymbol{\epsilon}_{k+1}^{I}\right\| \leq C_{r s d}
$$

where $k$ and $k+1$ are iteration numbers and $C_{r s d}$ the critical residual.

\section{Instructive examples}
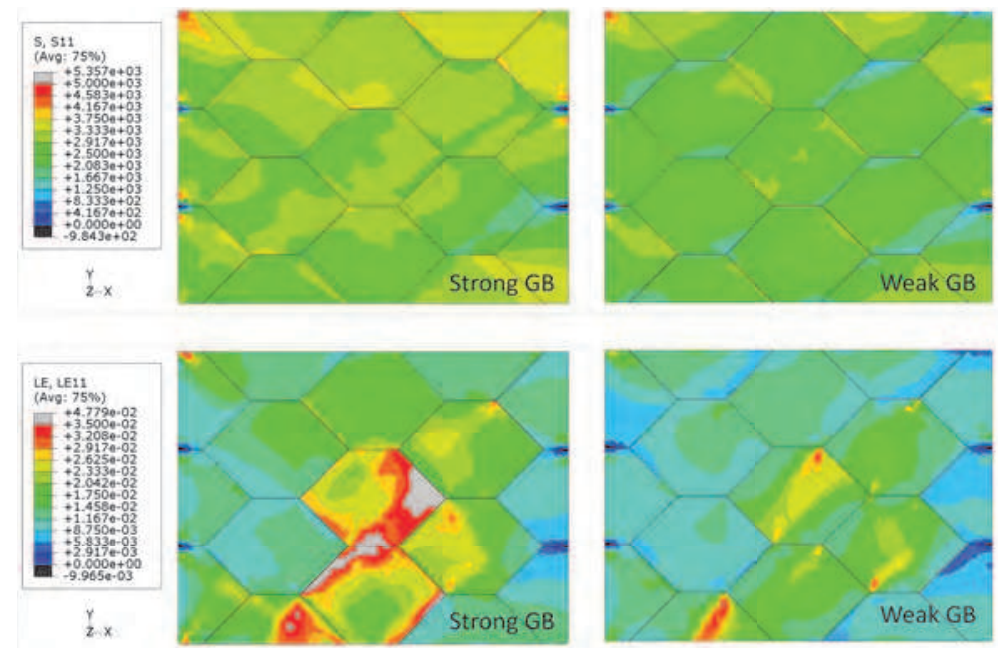

Fig. 6. Local stress and strain patterns of RVEs having grain boundaries with with different properties. 


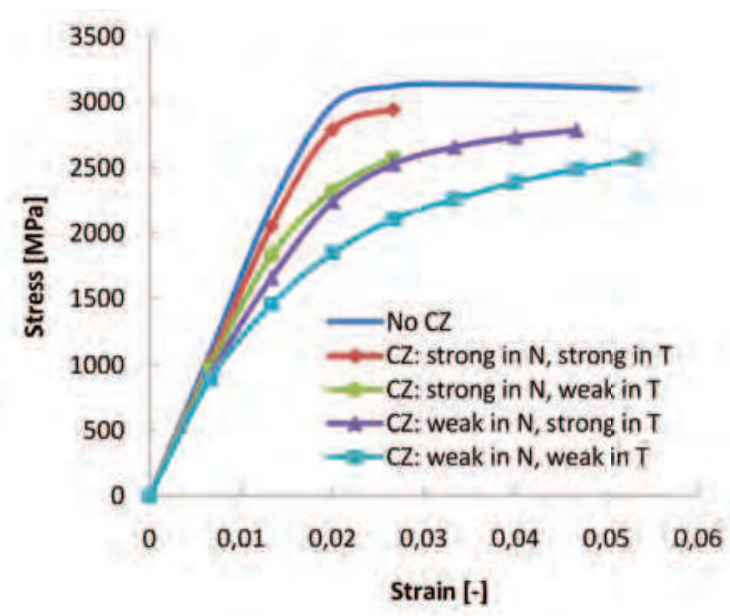

Fig. 7. Global stress-strain curves of RVEs having grain boundaries with with different properties.

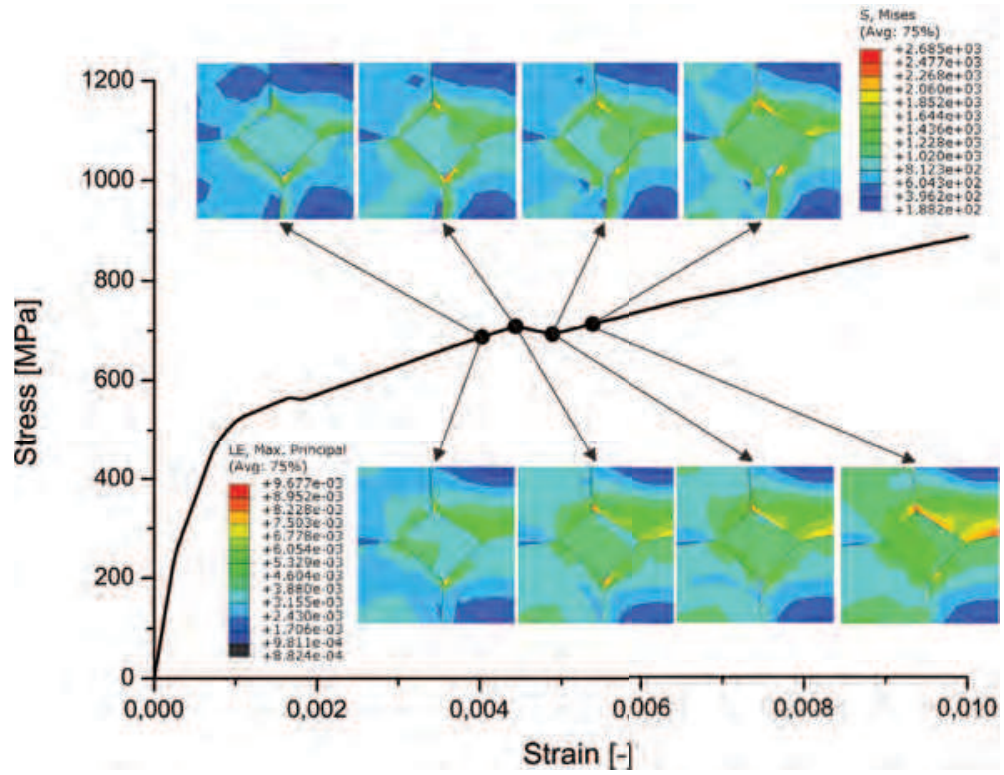

Fig. 8. Global stress-strain curve and local stress and strain distribution.

\subsection{Polycrystal deformation modelling with bulk material slip and grain boundary material glide with the crystal plasticity method}

A qusi-2D RVE with 17 hexagonal shape grains with $80 \mathrm{~nm} \times 60 \mathrm{~nm} \times 2 \mathrm{~nm}$ volume has been generated. Keeping $\phi=0$ and $\varphi_{2}=0$ we have assigned initial crystal orientations with an $5^{\circ}$ increment for Euler angle $\varphi_{1}$ from grain $0^{\circ}$ to $80^{\circ}$ randomly. For the studied aluminum 
polycrystal slip systems with $a / 2[1,1,0]$ and $a / 2[-1,1,0]$ Burgers vectors will be activated under tensile loads along the horizontal direction (Shaban et al., 2010).

Figure 6 shows the stress and strain distributions at about $2 \%$ tensile strain with a loading speed of $10 \mathrm{~nm} / \mathrm{s}$. These results are calculated with combinations of a normal interface strength of $1500 \mathrm{MPa}$ and two tangential interface strengths; an strong one of $1500 \mathrm{MPa}$ and an weak one of $300 \mathrm{MPa}$. One can easily see that the stronger grain boundary causes higher stress concentrations and strain heterogeneities inside the aggregate compared with the weaker grain boundary. For the weaker grain boundary, the strain localisation instead starts from the triple junction and tends to expand into the bulk material roughly along the maximum shear stress direction. Although this phenomenon is also observed for the stronger grain boundaries, the most obvious strain localisation is in some grains with a larger Schmid factor along the grain boundary direction and even extending to the grain center in some extreme cases. In both cases, cracks have initiated in the upper and lower boundaries and attempted to propagate along the vertical grain boundaries under tensile loading along the horizontal direction.

Figure 7 shows the global stress-strain curves with respect to 5 different grain boundary strength conditions. From this plot, one can see that the combination of grain boundary opening and sliding can relax almost one third of the average stress level. Because the weak-normal-strong-tangential cohesive zone model and strong-normal-weak-tangential cohesive zone model produce almost the same global stress strain curve, it seems that the normal and tangential cohesive strengths have similar influences on the material load carrying capacity.

With respect to an small qusi-2D RVE with 5 grains in a $1 \mu m \times 1 \mu m$ domain, the details of the global stress strain curve have been studied as shown in Figure 8. The RVE shows some unstable mechanical behaviors especially at about $0.5 \%$ tensile strain. Generally the material load carrying capacity loss infers the damage initialisation. From our calculations, one can see clearly that the kinks of the stress strain curve are mainly stemming from grain boundary opening and sliding near grain boundary triple junctions which can relax the locally accumulated stress efficiently.

The current study with respective to nano-metere grain size polycrystals implies that grain boundary mediated deformation processes decisively change the global stress-strain response of the studied material. Since the grain boundary cohesive behavior is independent of the grain size, whereas the resistance for dislocation slip inside the bulk material points becomes smaller as grain size increase, we expect that the influence of grain boundary processes will gradually vanish for coarse-grained material.

\subsection{TRIP steel deformation modelling with the crystal plasticity method}

An RVE including 12 ferritic grains and one austenitic grain as shown in Figure 9 has been generated to investigate the TRIP behavior under different loading conditions. As shown in Figure 10, tensile and compression loadings induce different total martensitic volume fractions. This numerical result is consistent with experimental observations. During the martensite phase transformation, about $22 \%$ shear strain inside the habit plane and about $2 \%$ dilatation strain along the normal of the habit plane are needed to transfer from the FCC 


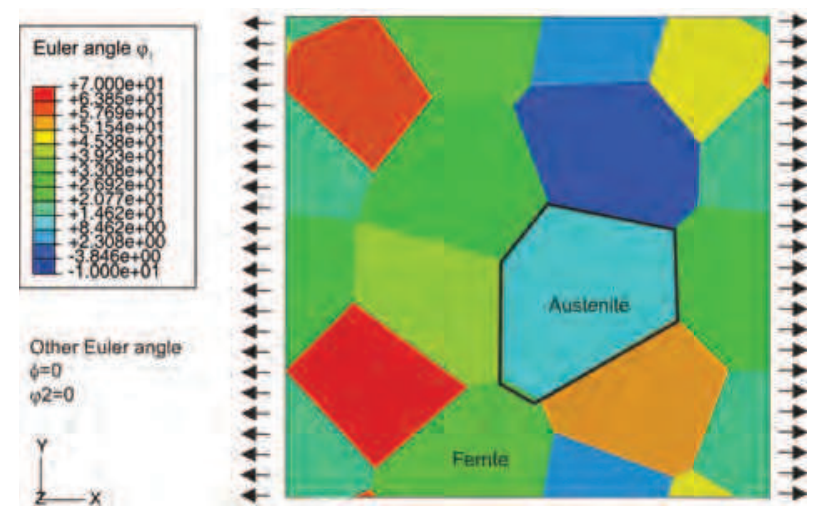

Fig. 9. Initial orientations of ferritic and austenitic grains. The only austenitic grain has been highlighted and has a volume fraction of about $10 \%$. For the compression calculation, the loading direction has been inversed.

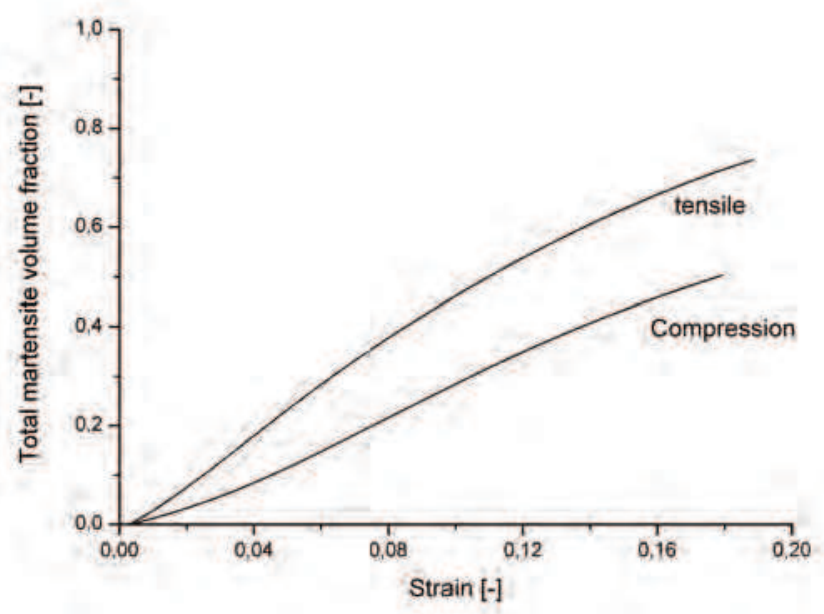

Fig. 10. Total martensitic volume fractions under tensile and compression loading cases.

lattice to the BCT lattice. Modelling results support that the normal part of the resolved stress calculated by equation 37 strongly influences the transformation volume fraction evolution. As stated in literature (Kouznetsova \& Geers, 2008; Stringfellow et al., 1992), this is the well known hydrostatic stress dependence of the martensite transformation. The current study shows that there are four dominating transformation systems under tensile and compression loading conditions as shown in Figure 11. Careful analysis of the magnitude of dilatation and shear resolved stresses under tensile and compression loads shows that the shear part is important to determine the activated transformation systems. 


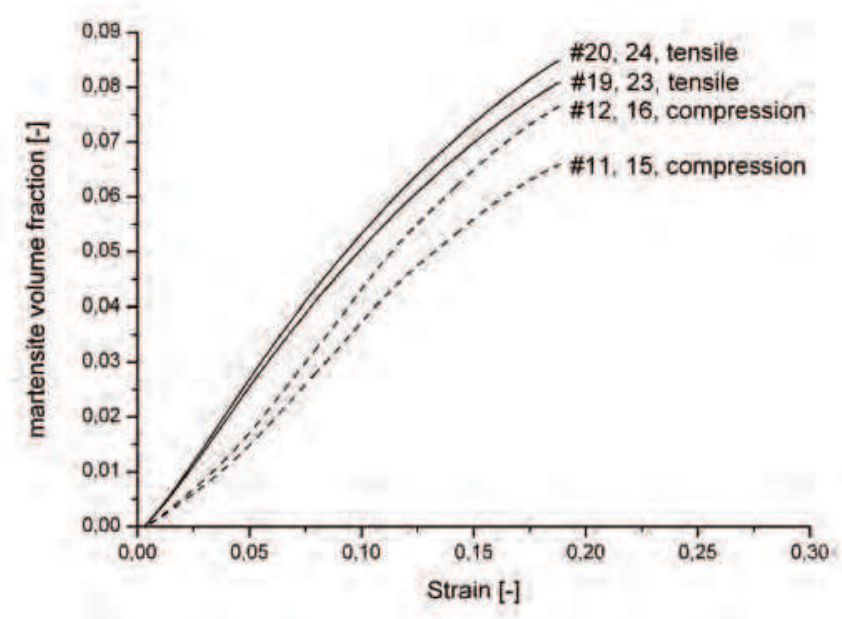

Fig. 11. Martensitic volume fractions of specific transformation systems under tensile and compression loads.

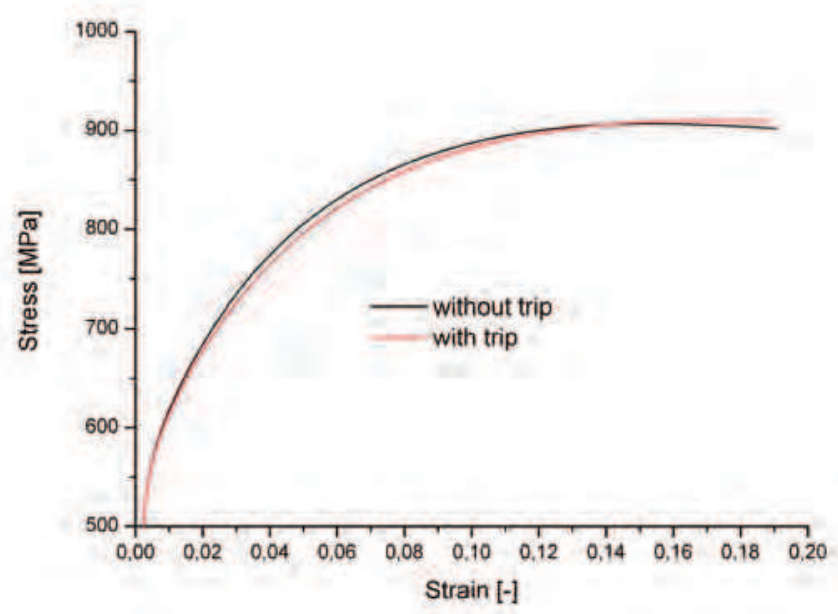

Fig. 12. Stress-strain curve comparison between deformations with and without martensite transformation.

With the help of the 13 grain RVE we have investigated into why martensite phase transformation can provide high ductility and high strength at the same time. As shown in Figure 12, the global stress-strain curves of the simulation with and without martensite transformation have a intersection point at about $14 \%$ tensile strain. Before the intersection point, the eigen strain of the phase transformation serves as a competing partner of dislocation 

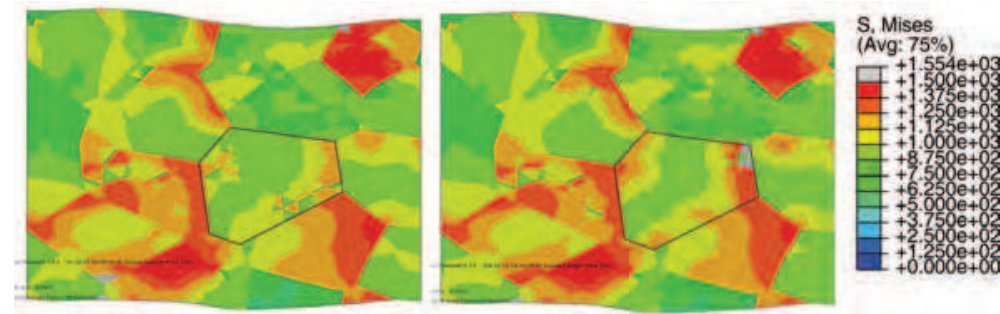

Fig. 13. Local stress distribution comparison between deformations with (right) and without (left) martensite transformation. The austenitic grain has been highlighted.

slip to reduce the external load potential, and as a direct result TRIP can increase the material ductility as shown in Figure 12. Because the phase transformation will exhaust the dislocation slip volume fraction and the martensite can only deform elastically, after the intersection point the hardening side of the TRIP mechanism will overcome the softening side and one can observe there is a enhanced tensile strength.

Figure 13 shows the local stress distribution comparison between simulations with and without phase transformation. As expected there are higher internal stresses inside the austenite grain when phase transformation exists. Through modelling the internal stress accumulation the current model system can be used to investigate the material damage and failure phenomena.
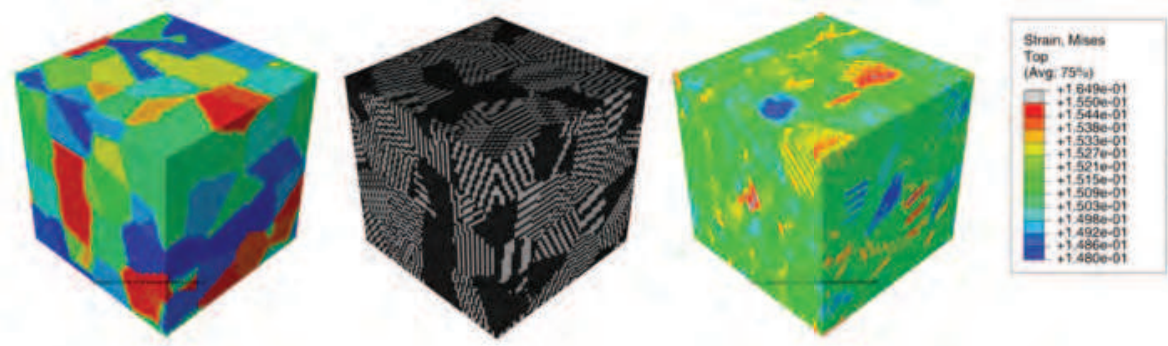

Fig. 14. Local strain distributions of an 189 grain RVE.

\subsection{Polycrystal with twin lamella deformation modelling with the crystal plasticity method}

As a mesh free method, the fast Fourier transformation approach can be used to model deformation of RVEs with very complicated microstructures. Several RVEs occupying a $1 \mu \mathrm{m} \times 1 \mu \mathrm{m} \times 1 \mu \mathrm{m}$ space with nano-metere sized twin lamellas inside nano-metere sized grains have been generated and discretised to $64 \times 64 \times 64$ regular grids. The initial crystal orientations have been assigned randomly. Based on equations 5 and 6, twin lamellas with different thickness have been generated. Figure 14 shows an RVE with 189 grains containing a $31 \mathrm{~nm}$ thickness lamella and the von-Mises equivlent strain distribution under tensile load and periodic boundary conditions. Here, the material parameters of pure aluminum have been used during the simulation. 


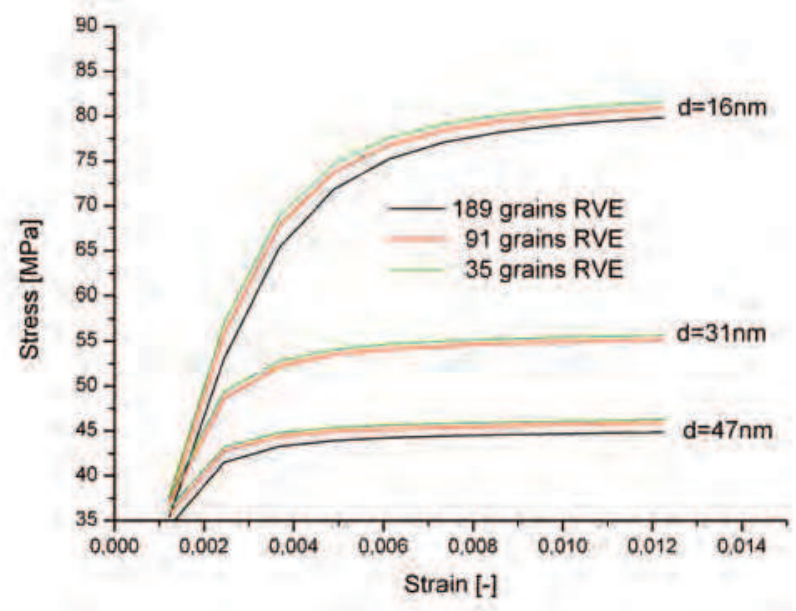

Fig. 15. Stress-strain curve comparison among RVEs with different grain size and lamella thickness.

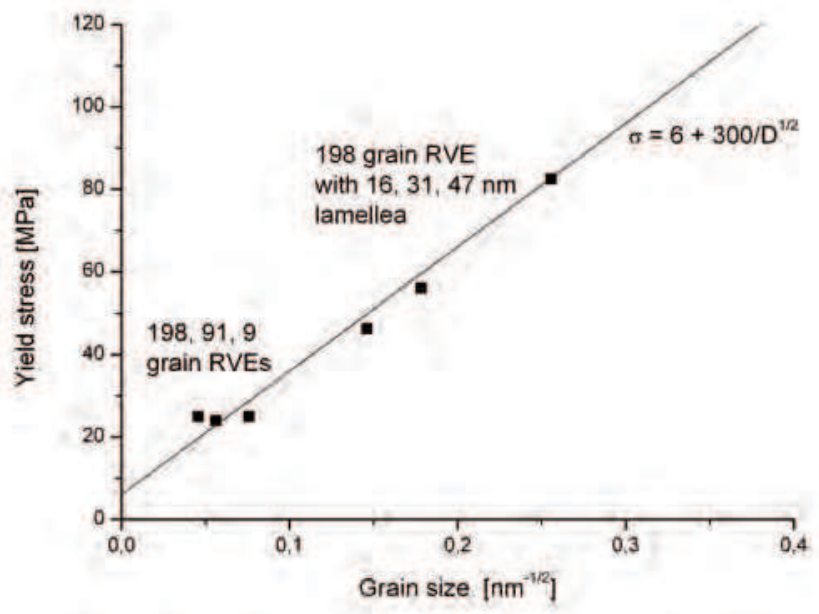

Fig. 16. Yield stress comparison among RVEs with different grain size and lamella thickness.

With respect to four grain numbers $(189,91,35,9)$ and three lamella thicknesses $(16 \mathrm{~nm}, 31$ $\mathrm{nm}, 47 \mathrm{~nm}$ ), a total of 12 global stress-strain curves have been simulated to investigate the size effect on material mechanical behaviors. Figure 15 shows several stress-strain curves along the loading direction. From these results one can see easily the smaller the stronger rule often observed in experiments. Through determining the yield stress for different RVEs, the 
parameters $\sigma_{0}$ and $k_{y}$ of the Hall-Petch relation

$$
\sigma=\sigma_{0}+\frac{k_{y}}{\sqrt{D}}
$$

for pure aluminum have been investigated carefully. From the numerical results shown in Figure 16 we found that equation 60 with parameters $\sigma_{0}=6 \mathrm{MPa}$ and $k_{y}=300 \mathrm{MPa} \sqrt{n m}$ fits the simulation data very well. Indeed, these two values almost fall nicely in the experimental measurement ranges $\sigma_{0}=6 \pm 2 \mathrm{MPa}$ and $k_{y}=400 \pm 80 \mathrm{MPa} \sqrt{n m}$ in Bonetti et al. (1992).

\section{Summary}

In this work it has been demonstrated how information from several length scales can be integrated into representative volume element (RVE) models for the mechanical behaviour of heterogeneous materials, consisting of several grains and different phases. In particular, the relevance of phenomena on different scales, like atomic bonds that determine the mechanical properties of grain boundaries, or the interaction of dislocations with grain boundaries should be investigated carefully in future studies. The mechanisms occurring at such atomistic and microstructural scales need to be modelled in a suited way such that they can be taken into account in continuum simulations of RVE's. Once an RVE for a given microstructure is constructed and the critical deformation and damage mechanisms are included into the constitutive relations, this RVE can be applied to calculate stress-strain curves and other mechanical data. The advantage of this approach is that by conducting parametric studies the influence of several microstructural features, like for example grain size or strength of grain boundaries, on the macroscopic mechanical response of a material can be predicted.

\section{References}

ABAQUS (2009). ABAQUS Version6.91, Dassault Systemes.

Bhadeshia, K. (2002). Trip-assisted steels?, ISIJ International 42: 1059.

Bhattacharya, K. (1993). Comparison of the geometrically nonlinear and linear theories of martensitic-transformation, Continuum Mech. Therm. 5: 205.

Bonetti, E., Pasquini, L. \& Sampaolesi, E. (1992). The influence of grain size on the mechanical properties of nanocrystalline aluminium, Nanostructured Materials 9: 611.

Dai, H. \& Parks, D. (1997). Geometrically-necessary dislocation density and scale-dependent crystal plasticity, Khan, A., (Ed.),Proceedings of Sixth International Symposium on Plasticity, Gordon and Breach .

Devincre, B., Hoc, T. \& Kubin, L. (2008). Dislocation mean free paths and strain hardening of crystals, Science 320: 1745.

Gottstein, G. (2004). Physical Foundations of Materials Science, Springer Verlag, Berlin-Heidelberg, Germany.

Hane, K. \& Shield, T. (1998). Symmetry and microstructure in martensites, Philosophical Magazine A 78.

Hane, K. \& Shield, T. (1999). Microstructure in the cubic to monoclinic transition in titanium-nickel shape memory alloys, Acta Mater. 47.

Hirth, J. \& Lothe, J. (1992). Theory of dislocations, Krieger Pub Co. 
Janisch, R., Ahmed, N. \& Hartmaier, A. (2010). Ab initio tensile tests of Al bulk crystals and grain boundaries: universality of mechanical behavior, Physical Review $B$ 81: 184108-1-6.

Kalidindi, S., Bronkhort, C. \& Anand, L. (1992). Crystallographic texture evolution in bulk deformation processing of fcc metals, J. Mech. Phys. Solids 40.

Kocks, U., Argon, A. \& Ashby, M. (1975). Thermodynamics and kinetics of slip, Chalmers, B., Christian, J.W., Massalski, T.B. (Eds.), Progress in Materials Science 19: 1-289.

Kouznetsova, V. \& Geers, M. (2008). A multi-scale model of martensitic transformation plasticity, Mechanics of Materials 40: 641.

Lebensohn, R. A. (2001). N-site modeling of a 3d viscoplastic polycrystal using fast fourier transform, Acta Materialia 49: 2723.

Lee, E. (1969). Elastic-plastic deformation at finite strains, J Appl. Mech. 36: 1-6.

Lu, K., Lu, L. \& Suresh, S. (2009). Strengthening materials by engineering coherent internal boundaries at the nanoscale, Science 324: 349.

Lu, L., Shen, Y., Chen, X., Qian, L. \& Lu, K. (2004). Ultrahigh strength and high electrical conductivity in copper, Science 304: 422.

Ma, A. (2006). Modeling the constitutive behavior of polycrystalline metals based on dislocation mechanisms, Phd thesis, RWTH Aachen.

Ma, A. \& Roters, F. (2004). A constitutive model for fcc single crystals based on dislocation densities and its application to uniaxial compression of aluminium single crystals, Acta Materialia 52: 3603-3612.

Madec, R., Devincre, B., Kubin, L., Hoc, T. \& Rodney, D. (2008). The role of collinear interaction in dislocation-induced hardening, Science 301: 1879.

Michel, J., Moulinec, H. \& Suquet, P. (2000). A computational method based on augmented lagrangians and fast fourier transforms for composites with high contrast, Comput. Model. Eng. Sci. 1: 79.

Michel, J., Moulinec, H. \& Suquet, P. (2001). A computational scheme for linear and non-linear composites with arbitrary phase contrast, Int. J. Numer. Methods Eng. 52: 139.

Nemat-Nasser, S., Luqun, N. \& Okinaka, T. (1998). A constitutive model for fcc crystals with application to polycrystalline ofhc copper, Mech. Mater. 30: 325-341.

Nye, J. (1953). Some geometrical relations in dislocated crystals, Acta Metall. 1: 153-162.

Olson, G. \& Cohen, M. (1972). A mechanism for strain-induced nucleation of martensitic transformations, J. Less-Common Metals 28.

Peirce, D., Asaro, R. \& Needleman, A. (1982). An analysis of non-uniform and localized deformation in ductile single crystals, Acta Metall. 30: 1087-1119.

Roters, F. (1999). Realisierung eines Mehrebenenkonzeptes in der Plastizitätsmodellierung, Phd thesis, RWTH Aachen.

Schröder, J., Balzani, D. \& Brands, D. (2010). Approximation of random microstructures by periodic statistically similar representative volume elements based on lineal-path functions, Archive of Applied Mechanics 81: 975.

Shaban, A., Ma, A. \& Hartmaier, A. (2010). Polycrystalline material deformation modeling with grain boundary sliding and damage accumulation, Proceedings of 18th European Conference on Fracture (ECF18) .

Stringfellow, R., Parks, D. \& Olson, G. (1992). A constitutive model for transformation plasticity accompanying strain-induced martensitic transformation in metastable austenitic steels, Acta Metall. Mater. 40: 1703. 
Tjahjanto, D. D. (2008). Micromechanical modeling and simulations of transformation-induced plasticity in multiphase carbon steels, Phd thesis, Delft University of Technology.

Wechsler, M., Lieberman, T. \& Read, T. (1953). On the theory of the formation of martensite, Trans. AIME J. Metals 197: 1094.

Wei, Y. \& Anand, L. (2004). Grain-boundary sliding and separation in polycrystalline metals: application to nanocrystalline fcc metals, Journal of the Mechanics and Physics of Solids 52: 2587. 


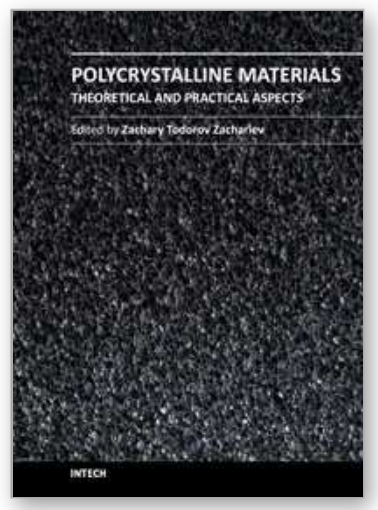

\author{
Polycrystalline Materials - Theoretical and Practical Aspects \\ Edited by Prof. Zaharii Zakhariev
}

ISBN 978-953-307-934-9

Hard cover, 164 pages

Publisher InTech

Published online 20, January, 2012

Published in print edition January, 2012

The book "Polycrystalline Materials - Theoretical and Practical Aspects" is focused on contemporary investigations of plastic deformation, strength and grain-scale approaches, methods of synthesis, structurals, properties, and application of some polycrystalline materials. It is intended for students, post-graduate students, and scientists in the field of polycrystalline materials.

\title{
How to reference
}

In order to correctly reference this scholarly work, feel free to copy and paste the following:

Anxin Ma and Alexander Hartmaier (2012). Scale Bridging Modeling of Plastic Deformation and Damage Initiation in Polycrystals, Polycrystalline Materials - Theoretical and Practical Aspects, Prof. Zaharii Zakhariev (Ed.), ISBN: 978-953-307-934-9, InTech, Available from: http://www.intechopen.com/books/polycrystallinematerials-theoretical-and-practical-aspects/scale-bridging-modelling-of-plastic-deformation-and-damageinitiation-in-polycrystals

\section{INTECH}

open science | open minds

\section{InTech Europe}

University Campus STeP Ri

Slavka Krautzeka 83/A

51000 Rijeka, Croatia

Phone: +385 (51) 770447

Fax: +385 (51) 686166

www.intechopen.com

\section{InTech China}

Unit 405, Office Block, Hotel Equatorial Shanghai

No.65, Yan An Road (West), Shanghai, 200040, China

中国上海市延安西路65号上海国际贵都大饭店办公楼 405 单元

Phone: +86-21-62489820

Fax: $+86-21-62489821$ 
(C) 2012 The Author(s). Licensee IntechOpen. This is an open access article distributed under the terms of the Creative Commons Attribution 3.0 License, which permits unrestricted use, distribution, and reproduction in any medium, provided the original work is properly cited. 